\title{
A Robust Method of Calculating the Effective Length of a Conductive Strip on an Ungrounded Dielectric Substrate
}

\author{
Manimaran Kanesan*, David V. Thiel, and Steve O'Keefe
}

\begin{abstract}
Dipole antennas on a substrate without a ground plane are common in wireless sensor networks and RFID applications. This paper reviews a number of theoretical approaches to solving for the effective permittivity when the substrate material is thin. The surface impedance and slab waveguide propagation techniques are compared to a capacitive solution and an insulated wire antenna. The insulated wire method gives most accurate results $(<3.5 \%)$ and was verified using numerical modeling and experimental work. Measurements on a planar straight dipole on FR4 $\left(f_{c}=1.50 \mathrm{GHz}\right)$ compare favorably with the antenna modeled without the substrate and scaled using the insulated wire technique at $\left(f_{c}=1.49 \mathrm{GHz}\right)$. The method can be readily incorporate the effect of an RFID antenna on a thin plastic film placed on a wide variety of lossy and lossless objects.
\end{abstract}

\section{INTRODUCTION}

Electrically small dipole antennas are commonly used in applications where size and cost are very important. For example, should RFID tags be used to replace optical bar codes on products in the supermarket, the number of antennas required to service this need would be greater than 1 billion units each year world-wide. In reducing the cost, the material cost should be minimal [1] and printed conductor technology can be used to minimize production costs. When a dipole length $L$ is placed on a substrate, its effective length is increased and the resonant frequency decreases. The increase in effective length depends on the electromagnetic properties of the substrate (commonly very thin plastic sheeting with an adhesive) and the material which supports the antenna substrate $[2-5]$. The change in the effective length depends on the width $w$ of the conducting strip, the permittivity $\varepsilon_{r}$ of the substrate, the thickness $d$ of the substrate and also the support material. Figure 1 illustrates these parameters for a printed dipole antenna.

While flexible dipole antennas are common, there is no simple method for calculating the effect of the substrate and the material on which the antenna is placed. Some researchers used optimization [6], a trial and error method [7], and computational methods [8]. These methods are very useful and approximate solutions can be obtained. Nonetheless these methods are time consuming, computationally difficult to solve, and required many computational iterations for each new antenna configuration. A coplanar strip (CPS) technique involves the calculation of the capacitance [3] but the solution for lower resonant frequencies on a thin substrate does not exist. If the gap $s$ between the two halves of the dipole is large, the capacitance approaches zero [2] and the results are inaccurate. As there is no conductive ground plane, a microstrip solution is not applicable and other similar structures (e.g., suspended, inverted, truncated and paired strips) are also not applicable.

The method of moments code NEC [9] can be used to calculate the resonant length of a wire dipole on a lossy substrate [4] and a method of converting a wire dipole to a planar structure on a dielectric substrate using the Hallen transformation [10] has been reported [2]. This paper describes the formulation of a number of approaches and compares their results with three dimensional FEM solutions and experimental results.

\footnotetext{
Received 24 December 2013, Accepted 25 February 2014, Scheduled 26 February 2014

* Corresponding author: Manimaran Kanesan (manimaran.kanesan@griffithuni.edu.au).

The authors are with the Griffith School of Engineering, Nathan Campus, Griffith University, Queensland, Australia.
} 


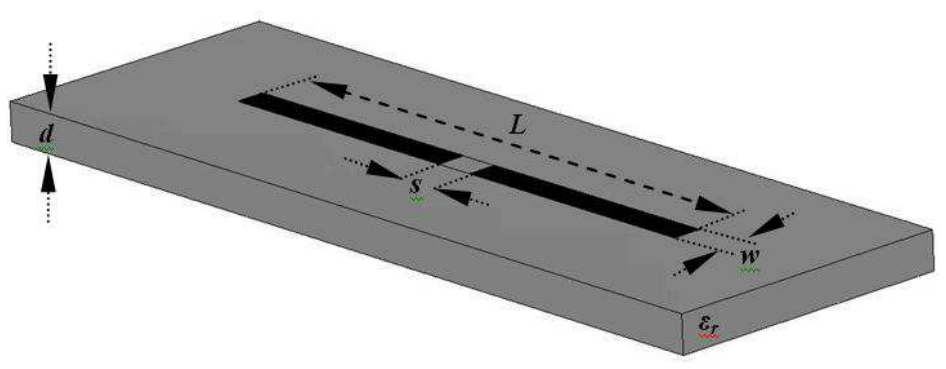

Figure 1. Planar dipole antenna on a dielectric substrate.

\section{THEORETICAL REVIEW}

The electromagnetic performance of a thin dielectric slab can be modeled using plane wave solutions. The electromagnetic performance of a conducting strip on a thin dielectric slab requires additional complexity. Four different solutions were investigated (a) surface impedance of an infinite layered half space (plane wave solution) [11], (b) dielectric slab waveguide propagation (trapped wave solution), (c) an insulated wire propagation mode (surface wave solution) [12] and (d) the CPS technique (quasistatic capacitance solution) described briefly [3]. The theories for (a), (b) and (c) have been used in the solution of various problems, but they have not been previously applied to a printed antenna on an insulating substrate. In all cases, the velocity of propagation of a TEM wave is modified by the presence of the dielectric layer. The effective wavelength is scaled by the square root of the effective permittivity which is the parameter used throughout this paper.

\subsection{Surface Impedance Formulation}

A plane wave normally incident on a layered material can be calculated using surface impedance theory. Wait [13] showed that the surface impedance of multiple horizontal layers can be solved using transmission line equations. Assume we have a layered medium in which an electromagnetic plane wave in air (medium 1, intrinsic impedance $\eta_{1}$ ) is incident on the horizontal plane (the dielectric substrate, medium 2, intrinsic impedance $\eta_{2}$ ) and the next layer in the structure is air (medium 3, intrinsic impedance $\eta_{3}$ ). This is shown in Figure 2 .

For a loss-less dielectric, the surface impedance $Z_{\text {in }}$ can be written as

$$
Z_{\text {in }}=\eta_{2} \frac{\eta_{3}+j \eta_{2} \tan (\beta d)}{\eta_{2}+j \eta_{3} \tan (\beta d)}
$$

where $\eta_{1}=\eta_{3}=$ intrinsic impedance of free space. The phase constant in the dielectric, $\beta$ is given by,

$$
\beta=\frac{2 \pi \sqrt{\varepsilon_{r}}}{\lambda}
$$

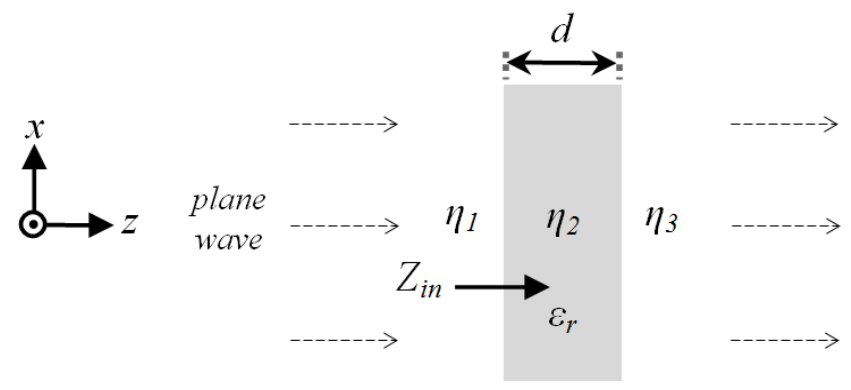

Figure 2. Plane wave normally incident on a thin dielectric substrate. 
and $\eta_{2}$ is,

$$
\eta_{2}=\sqrt{\frac{\mu}{\varepsilon}}=\sqrt{\frac{\mu_{0} \mu_{r}}{\varepsilon_{0} \varepsilon_{r}}}=\frac{\eta_{0}}{\sqrt{\varepsilon_{r}}}
$$

where $\varepsilon_{0}$ and $\mu_{0}$ are free space permittivity and permeability, and $\varepsilon_{r}$ and $\mu_{r}$ are the relative permittivity and permeability, respectively. The substrate relative magnetic permeability is assumed to be $\mu_{r}=1$.

By writing,

$$
Z_{\text {in }}=\sqrt{\frac{\mu_{0}}{\varepsilon^{\prime}+\varepsilon^{\prime \prime}}}
$$

then $\beta$ for surface impedance $\beta_{S I}$ can be written as [11]:

$$
\beta_{S I}=\omega\left\{\frac{\mu \varepsilon^{\prime}}{2}\left(\sqrt{1+\left(\frac{\varepsilon^{\prime \prime}}{\varepsilon^{\prime}}\right)^{2}}+1\right)\right\}^{\frac{1}{2}}
$$

From $(5) \varepsilon^{\prime}$ and $\varepsilon^{\prime \prime}$ are the real and imaginary parts of the effective permittivity of the substrate. The effective relative permittivity from surface impedance solution $\sqrt{\varepsilon_{\text {effSI }}}$ for dielectric for different thickness and permittivity can be found:

$$
\sqrt{\varepsilon_{e f f S I}}=\frac{\operatorname{Re}\left(\beta_{S I}\right) \lambda}{2 \pi}
$$

where $\sqrt{\varepsilon_{e f f S I}}$ is a real number.

\subsection{Dielectric Slab Waveguide}

An infinitely large, dielectric slab waveguide supports surface waves and the $\mathrm{TM}_{0}$ mode is the fundamental propagation mode found in the dielectric slab of Figure 3 [14]. The EM wave is predominantly confined to the slab waveguide and propagates through the slab in the $z$ direction.

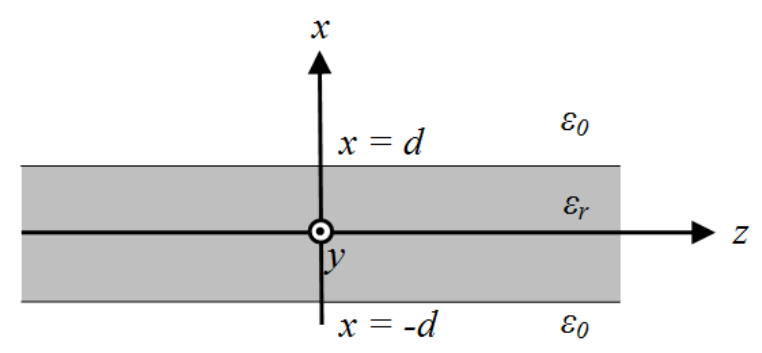

Figure 3. Geometry of a dielectric slab waveguide.

The relative effective permittivity for the slab waveguide $\sqrt{\varepsilon_{\text {effSlab }}}$ can be solved by calculating the constants $k_{c}$ and $h$ from the wave Equations (7) and (8) [14]. Here $k_{c}$ and $h$ are wavenumbers characterized in the homogenous dielectric and in air regions.

$$
\begin{aligned}
& \frac{\partial^{2} e_{z}(x, y)}{\partial x^{2}}+k_{c}^{2} e_{z}(x, y)=0 \\
& \frac{\partial^{2} e_{z}(x, y)}{\partial x^{2}}-h^{2} e_{z}(x, y)=0
\end{aligned}
$$

The cutoff wavenumber $k_{c}$ is given by [12],

$$
k_{c}=\sqrt{\varepsilon_{r} k_{0}^{2}-\beta_{S l a b}^{2}}
$$

where $k_{0}$ is free space wave number,

$$
h=\sqrt{\beta_{\text {Slab }}^{2}-k_{0}^{2}}
$$


Eliminating $\beta_{\text {slab }}$ from (9) and (10) gives,

$$
k_{c}^{2}+h^{2}=\left(\varepsilon_{r}-1\right) k_{0}^{2}
$$

Equation (11) is a transcendental equation where $k_{o}$ and $\varepsilon_{r}$ must be solved for propagation constants $k_{c}$ and $h$ by numerical techniques. Satisfying the boundary conditions (7) and (8), the nontrivial solution gives (12). By multiplying both sides by $d$ with correct $k_{c}$ and $h$ from (11) we have,

$$
k_{c} d \tan k_{c} d=\varepsilon_{r} h d
$$

This leads the correct $\beta$ for the slab waveguide, $\beta_{\text {slab }}$. From (6) and (12) $\sqrt{\varepsilon_{\text {effSlab }}}$ can be found from $\beta_{\text {slab }}$ for different dielectric thickness.

\subsection{Insulated Wire}

The presence of a thin insulated coating on a thin wire reduces $f_{c}$ due to the presence of surface wave. An insulated wire is similar to a dielectric slab on top of a conducting plane. The TM solution is possible with a thin material coating [15]. The insulated wire with radius wire $a$, radius dielectric coating $b$ and dielectric thickness $b-a=d$ is illustrated in Figure 4.

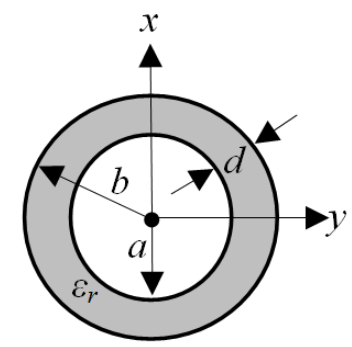

Figure 4. Conducting wire (radius $a$ ) with a thin dielectric coating (thickness $d$ ).

The $\beta$ for the insulated wire $\beta_{\text {wire }}$ is solvable from the transcendental Equation (13) involving Bessel functions. The wavenumbers $k_{c}$ and $h$ are similar to (9) and (10).

$$
\frac{K_{1}(h b)}{h K_{0}(h b)}=\frac{\varepsilon_{r}}{k_{c}} \frac{J_{0}\left(k_{c} a\right) Y_{1}\left(k_{c} b\right)-J_{1}\left(k_{c} b\right) Y_{0}\left(k_{c} a\right)}{J_{0}\left(k_{c} b\right) Y_{0}\left(k_{c} a\right)-J_{0}\left(k_{c} a\right) Y_{0}\left(k_{c} b\right)}
$$

where $K_{0}$ and $K_{1}$ are modified Bessel functions of second kind; $J_{0}$ and $J_{1}$ are Bessel functions of first kind; $Y_{0}$ and $Y_{1}$ are Bessel functions of second kind. The $\sqrt{\varepsilon_{\text {effWire }}}$ then can be found from (6). The solution for $\beta_{\text {Wire }}$ was calculated using $a=0.05 \mathrm{~mm}$. This is a requirements compromise between an infinitely thin wire and the discretization limits of the FEM voxel requirement.

\subsection{Coplanar Strips}

The technique used to obtain $\sqrt{\varepsilon_{e f f}}$ from [3] is based on a capacitance method using coplanar strips and transformed to the center fed dipole structure shown in Figure 1. The CPS structure consists of two planar conductors placed side by side and distanced by $s$ on an ungrounded substrate of $d$. To obtain the appropriate $\sqrt{\varepsilon_{e f f}}$ the capacitance of CPS structure is determined. The total effective capacitance of the CPS due to the electric field above the structure, in the structure and below the structure leads to the final effective capacitance of

$$
C_{\text {eff }}=\varepsilon_{o} w\left[2 K_{1}+\left(\varepsilon_{r}-1\right) K_{2}\right]
$$

where $K_{1}$ and $K_{2}$ are obtained from (15) and (16),

$$
\begin{aligned}
& K_{1}=\frac{K\left(k_{1}\right)}{K^{\prime}\left(k_{1}\right)} \\
& K_{2}=\frac{K\left(k_{2}\right)}{K^{\prime}\left(k_{2}\right)}
\end{aligned}
$$


and $K\left(k_{1}\right)$ and $K^{\prime}\left(k_{1}\right)$ is complete elliptical integral of first kind and its complimentary and $K\left(k_{2}\right)$ and $K^{\prime}\left(k_{2}\right)$ is complete elliptical integral of second kind and its complimentary. Using conformal mapping transformation and relating $k_{1}$ and $k_{2}$ to center fed dipole structure then the following equation obtained

$$
\begin{aligned}
& k_{1}=\frac{L-s}{L+s} \\
& k_{2}=\frac{\sinh \left(\frac{\pi(L-s)}{4 d}\right)}{\sinh \left(\frac{\pi(L+s)}{4 d}\right)}
\end{aligned}
$$

Then $\varepsilon_{\text {eff }}$ for the centre fed dipole antenna using CPS technique is determined from

$$
\varepsilon_{e f f C P S}=1+\left(\frac{\varepsilon_{r}-1}{2}\right) \frac{K_{2}}{K_{1}}
$$

The results for these different solutions, $\sqrt{\varepsilon_{\text {effSI }}}, \sqrt{\varepsilon_{\text {effSlab }}}, \sqrt{\varepsilon_{\text {effWire }}}$ for the fundamental mode and $\sqrt{\varepsilon_{\text {eff } C P S}}$ on a dielectric layer are bound by limits in $(20)$,

$$
1<\sqrt{\varepsilon_{\text {eff }}}<\sqrt{\varepsilon_{r}}
$$

Thus $\sqrt{\varepsilon_{\text {eff }}}$ will have values between free space to $\sqrt{\varepsilon_{r}}$ when $d=0$ to $d=\infty$ for CPS solution and for the fundamental propagation modes. This will not be the case for higher order modes. For practical reasons, most common planar antennas $d$ will lie in the range between $0.1 \mathrm{~mm}$ to $0.5 \mathrm{~mm}$ for plastic substrates, and $d=1.6 \mathrm{~mm}$ for the most common FR4 substrate. The $\sqrt{\varepsilon_{\text {eff }}}$ for all four solutions are plotted in Figure 5 together with FEM solution (discussed in Numerical Modeling section).

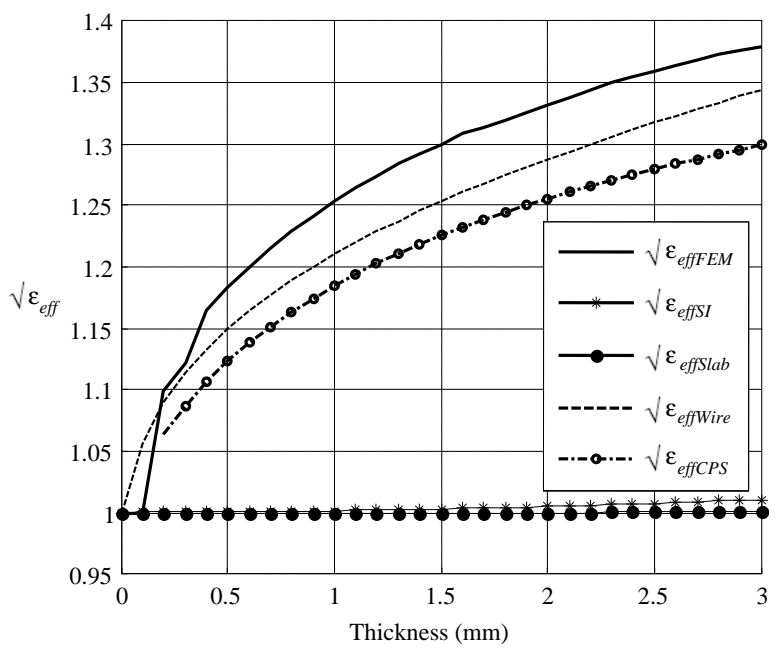

Figure 5. $\sqrt{\varepsilon_{\text {eff }}}$ plotted as for various calculation methods: FEM, surface impedance, dielectric slab waveguide, insulated wire, and CPS.

\section{NUMERICAL MODELING}

The half-wavelength dipole wire antenna is designed and transformed to a planar antenna using Hallen's transformation from $(21)$

$$
w=4 a
$$

A free space wire dipole with $a=0.25 \mathrm{~mm}$ and $L=154.2 \mathrm{~mm}$ resonating at $905.5 \mathrm{MHz}$ is transformed to dipole strip antenna (DSA) in free space using Equation (21). The width of the dipole $w=1 \mathrm{~mm}$ with $L$ equivalent to wire dipole has a similar resonant frequency to wire dipole. This resonance is the centre frequency for an RFID antenna operating in the frequency band: $860 \mathrm{MHz}$ to $960 \mathrm{MHz}$ [1]. The DSA 
in free space attached to a substrate (Figure 1) has the dipole antenna on top of the dielectric (relative permittivity $\varepsilon_{r}$ ) with source $s$ spaced at $1 \mathrm{~mm}$ in the center and distinguished as dipole planar antenna (DPA). The DPA with $\varepsilon_{r}=4.5$ was simulated using a 3D electromagnetic finite element method, FEM, for a range of thickness values $0 \mathrm{~mm}<d<3 \mathrm{~mm}$. The effective permittivity $\varepsilon_{\text {eff }}$ was calculated using:

$$
f_{c}=\frac{c}{2 L \sqrt{\varepsilon_{e f f}}}
$$

where $c$ is the free space velocity of light and $f_{c}$ the fundamental resonant frequency. For FEM calculations the effective relative permittivity is labeled as $\varepsilon_{\text {effFEM }}$.

The change in the resonant frequency $f_{c}$ and the effective relative permittivity $\varepsilon_{\text {eff }}$ (plotted as the square root of the effective permittivity) are given in Figure 6 . An increase in $d$ reduces $f_{c}$ for a fixed length $L$. An increase in $\varepsilon_{r}$, reduces $f_{c}$ and so $\sqrt{\varepsilon_{\text {eff }}}$ increases. When $d=0$, then $\sqrt{\varepsilon_{\text {eff }}}=1$.

This paper reports a method to calculate the antenna fundamental resonant frequency $f_{c}$ on an ungrounded substrate for thin substrates with various permittivity values.

\section{MODEL COMPARISON}

The calculated $\sqrt{\varepsilon_{\text {eff }}}$ are plotted in Figure 5 for $\varepsilon_{r}=4.5$ and $d=0$ to $3 \mathrm{~mm}$. Figure 5 shows the calculated solutions for $\sqrt{\varepsilon_{e f f}}$ all increase with increasing $d$ as demonstrated by $\sqrt{\varepsilon_{e f f F E M}}$. For all calculation methods $\sqrt{\varepsilon_{\text {eff }}}=1$ when $d=0$ showing good agreement to the FEM results $\sqrt{\varepsilon_{\text {effFEM }}}$. Note that a solution for $d=0$ and $0.1 \mathrm{~mm}$ does not exist for the CPS technique. It is clear, however, that the surface impedance method and the slab waveguide method do not show good agreement with the FEM results. This is thought to be because the antenna is strongly influenced by the quasi-static near fields of the antenna, and these two methods are far field results which do not include quasi-static effects. possible.

As all of the $\sqrt{\varepsilon_{e f f}}$ calculations are approximations, an exact match to the FEM results is not

The resonant frequency of the dipole $f_{c}$ for $d=0$ to $3 \mathrm{~mm}$ calculated using the four different solution methods are plotted in Figure 7 together with the FEM results. The plot shows that both $\sqrt{\varepsilon_{\text {effSI }}}$ and $\sqrt{\varepsilon_{\text {effSlab }}}$ has less significant change in $f_{c}$, and $\sqrt{\varepsilon_{\text {effSlab }}}$ has little change compared to $\sqrt{\varepsilon_{\text {effSI }}}$ when $d$ increases. This results in a maximum difference of $216 \mathrm{MHz}$ when $d=3 \mathrm{~mm}$ from the simulated $f_{c}$ (approximately $32 \%$ error). The CPS and insulated wire technique are promising methods to use. CPS has a $6 \%$ error with $43 \mathrm{MHz}$ maximum difference from the simulated $f_{c}$. The maximum insulated wire difference is $18 \mathrm{MHz}$ with approximately $3.5 \%$ error and less than $1 \%$ error for $d<0.3 \mathrm{~mm}$.

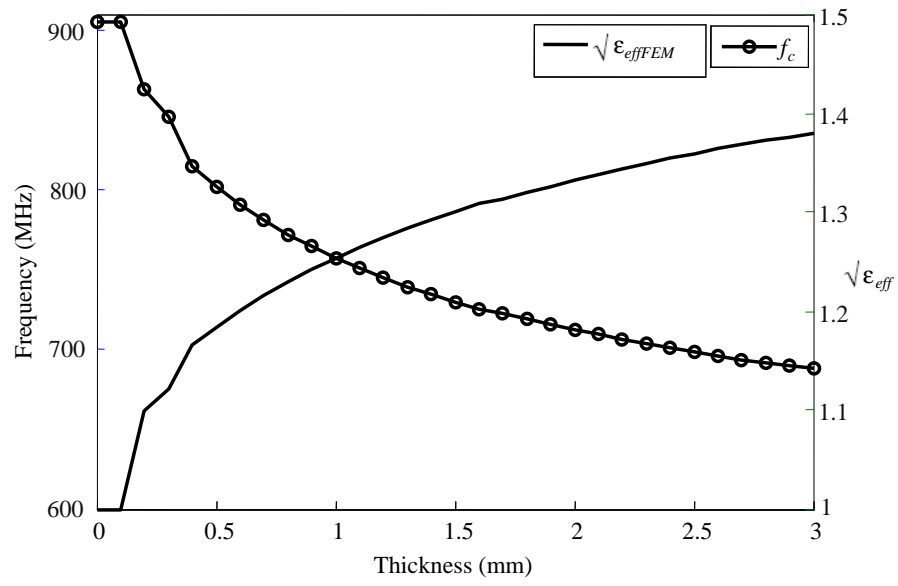

Figure 6. The DPA $f_{c}$ and $\sqrt{\varepsilon_{\text {eff }}}$ varies with the substrate thickness $d$. When $d=0$ then the DPA is in free space and the $\varepsilon_{\text {eff }}=1$.

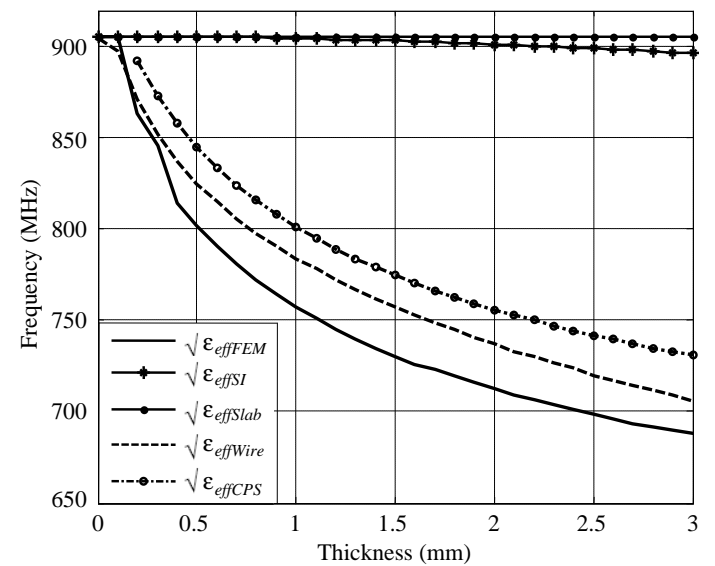

Figure 7. The resonant frequency $f_{c}$ for a dipole antenna on a slab dielectric (thickness $d$ ) for different calculation techniques for a dipole antenna on a dielectric substrate $\left(\varepsilon_{r}=4.5\right)$. 
As the RFID main operation bands are $433 \mathrm{MHz}, 860 \mathrm{MHz}$ to $960 \mathrm{MHz}, 2.45 \mathrm{GHz}$ and $5.8 \mathrm{GHz}$, the precision of the insulated wire technique was investigated across the frequency range $400 \mathrm{MHz}$ to $6 \mathrm{GHz}$ (Figures 8(a)-(d)) The DPA with $w=1 \mathrm{~mm}$ and $2 \mathrm{~mm}$ is included in Figure 8. Although $w$ has only a small effect on $f_{c}$, changing $w$ by $100 \%$ only change $f_{c}$ by $5 \%$ [3].

Figure 8 shows the insulated wire method is the better solution across all of the frequency bands considered. Although $f_{c}$ is similar at lower frequencies and the difference between these two solutions is small, CPS does not support thin substrates. At higher $f_{c}<3 \mathrm{GHz}$ the wire technique is more accurate with $<5.5 \%$ error compared to approximately $11 \%$ error for CPS and at $6 \mathrm{GHz}$ the wire technique is $<8 \%$ error compared to CPS with approximately 15\% error compared to the FEM modeling (and discussed in [3]).

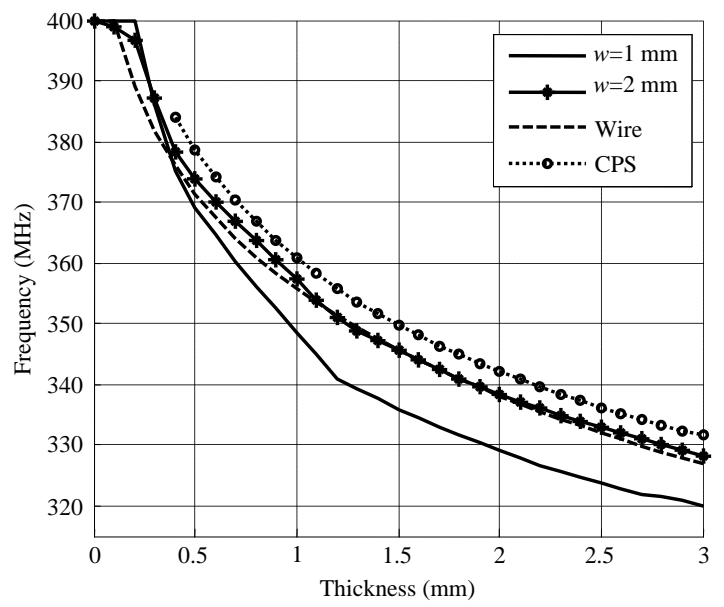

(a)

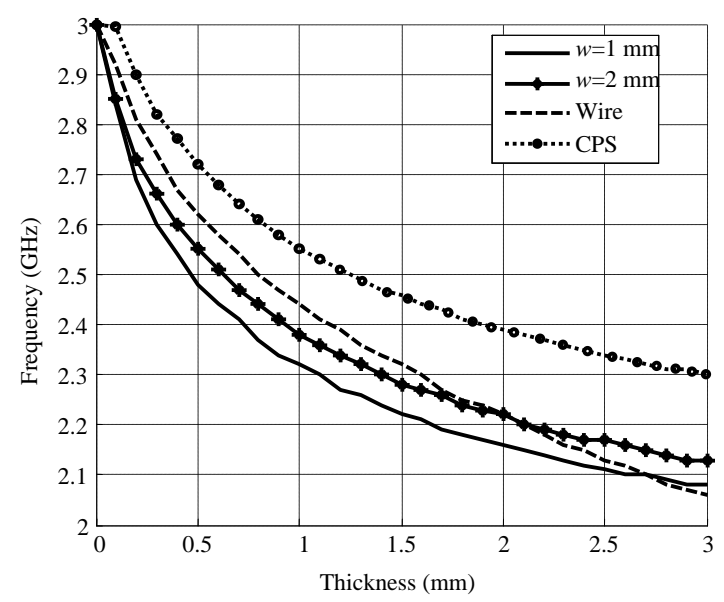

(c)

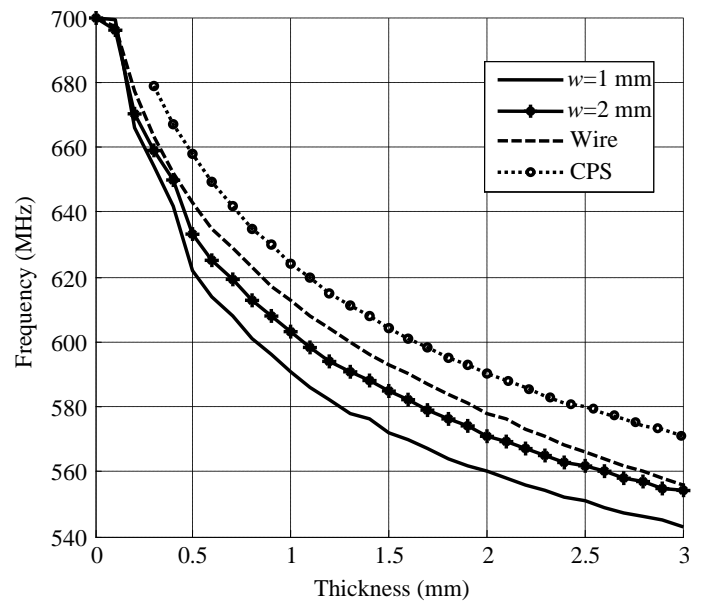

(b)

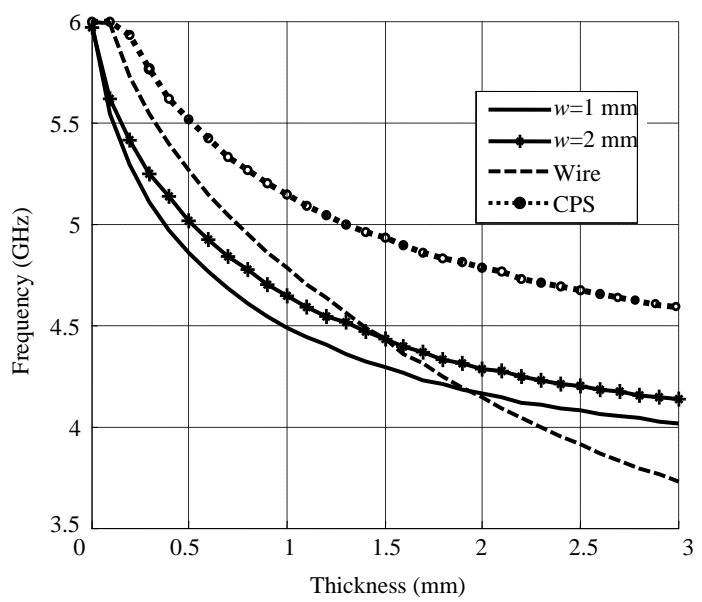

(d)

Figure 8. The variation between the FEM $f_{c}(w=1 \mathrm{~mm}$ and $2 \mathrm{~mm})$ and calculated method using the insulated wire antenna technique and CPS method for different frequency bands for: (a) $400 \mathrm{MHz}$, (b) $700 \mathrm{MHz}$, (c) $3 \mathrm{GHz}$, and (d) $6 \mathrm{GHz}$.

\section{EXPERIMENTAL MEASUREMENTS}

The $\sqrt{\varepsilon_{\text {effWire }}}$ solution was investigated using a dipole in air with $L=93.8 \mathrm{~mm}$ and $w=2 \mathrm{~mm}$ resonating at $f_{c}=1.5 \mathrm{GHz}$. A straight dipole of this size was fabricated on FR4 $\left(\varepsilon_{r}=4.5, d=1.6 \mathrm{~mm}\right.$ ) (Figure $9(\mathrm{a})$ ) and scaled back to $1.5 \mathrm{GHz}$ using the insulated wire method (Figure 9(b)). The resonant frequency $f_{c}$ of the DPA shifted to approximately $1.2 \mathrm{GHz}$ on the FR4 in simulation and was measured at $1.23 \mathrm{GHz}$. 


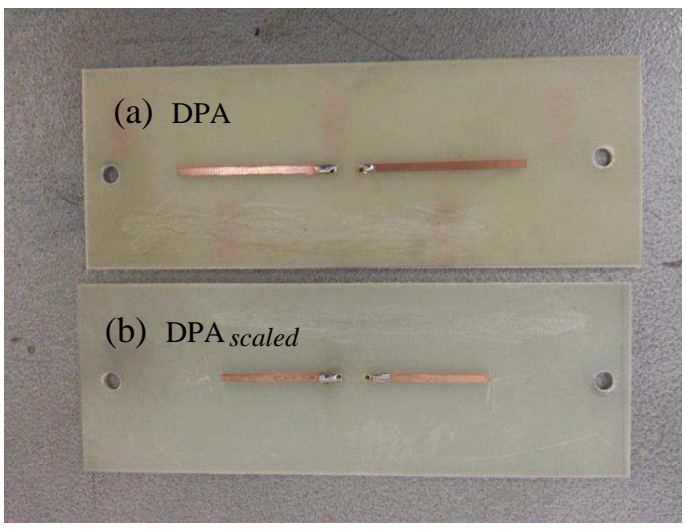

Figure 9. (a) A centre fed DPA $(L=93.8 \mathrm{~mm}$ and $w=2 \mathrm{~mm})$ was fabricated on $\mathrm{FR} 4\left(\varepsilon_{r}=4.5\right.$, $d=1.6 \mathrm{~mm})$. The DPA resonates at $1.23 \mathrm{GHz}$. (b) The DPA was scaled using $(23)\left(L_{\text {scale }}=72.5 \mathrm{~mm}\right)$ and the new resonant was $f_{c}$ at $1.49 \mathrm{GHz}$.

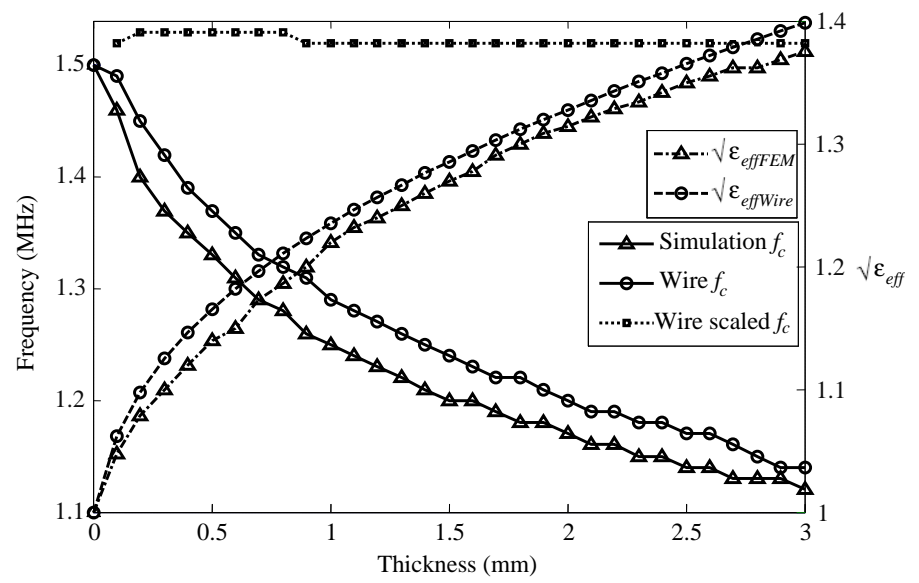

Figure 10. The resonant frequency of the DPA was calculated using the insulated wire technique is close to the FEM and the DPA scaled $_{\text {is approximately }}$ $1.525 \mathrm{GHz}$ for entire range of $d$ close to the $1.5 \mathrm{GHz}$ of desired $f_{c}$. In addition the corresponding effective permittivity is plotted as $\sqrt{\varepsilon_{e f f}}$.

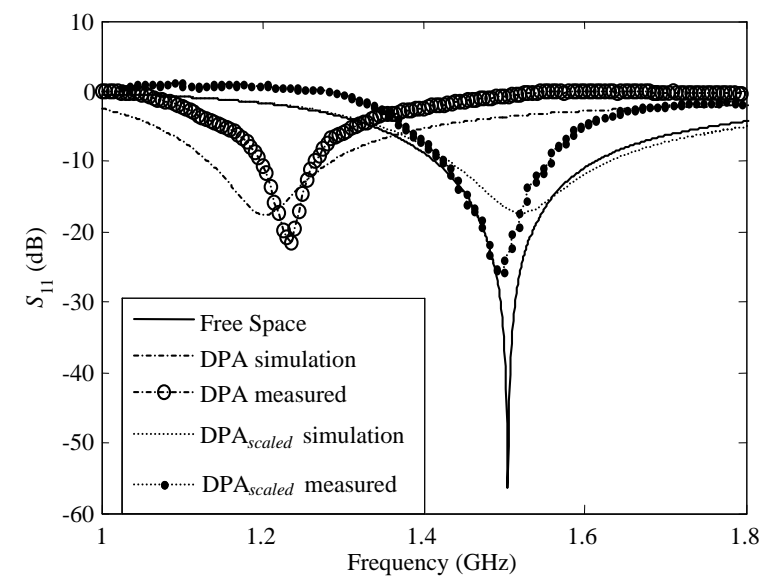

Figure 11. $S_{11}$ results for a $50 \Omega$ transmission showing the simulated and measured DPA, and $\mathrm{DPA}_{\text {scaled }}$ resonating at $1.49 \mathrm{GHz}$. The bandwidth is $9.3 \%$.

The solution of $\sqrt{\varepsilon_{\text {effWire }}}$ at $1.23 \mathrm{GHz}$ shows the insulated wire solution exhibits good agreement to the simulated and the measured $f_{c}$. The insulated wire solution and measured dipole are both within the $-10 \mathrm{~dB}$ bandwidth of FEM simulation result of $13 \%$.

The new antenna length was calculated without any optimization or trial and error method. To obtain the desired $f_{c}$ similar to DSA, the DPA length $L$ was scaled [2].

$$
L_{\text {scale }}=\frac{L}{\sqrt{\varepsilon_{\text {eff }}}}
$$

The scaling factor reduces the $L$ which increases $f_{c}$. The scaling approximations can be determined by $\sqrt{\varepsilon_{\text {effWire }}}$. Figure 10 illustrates the simulations results of scaling factor for $d=0$ to $3 \mathrm{~mm}$ where the DPA $L$ is scaled to the relative $d$ using $\sqrt{\varepsilon_{\text {effWire }}}$. The results show that the error is $<2 \%$, approximately $1.525 \mathrm{GHz}$ for entire range of $d$. In addition $\sqrt{\varepsilon_{\text {eff Wire }}}$ is very similar to $\sqrt{\varepsilon_{\text {effFEM }}}$. From Figure 11, the simulation for DPA scaled antenna in Figure 9 (b) resonates at $1.52 \mathrm{GHz}$ for insulated wire solution, and the measured antenna is resonating at $1.49 \mathrm{GHz}$ close to the desired frequency. The antenna $f_{c}$ is still within $-10 \mathrm{~dB}$ BW of the DSA, wire solution and measured $\mathrm{DPA}_{\text {scaled }}$. 


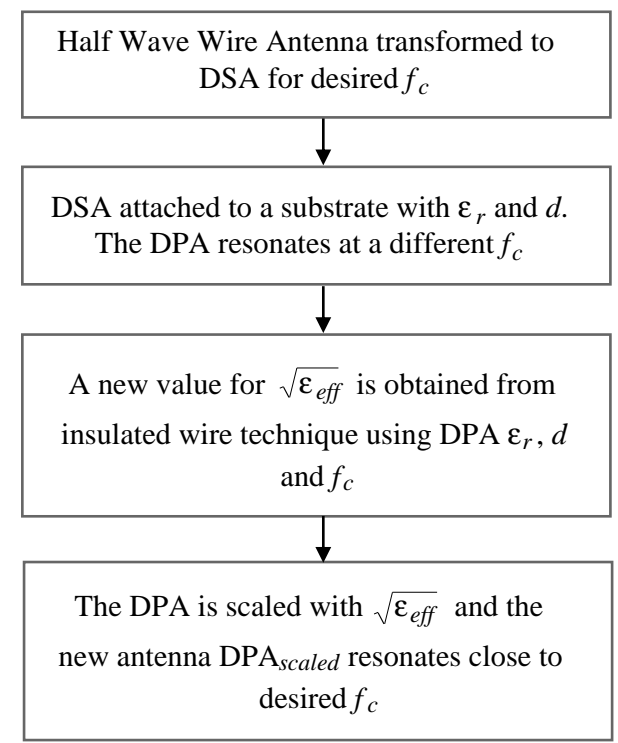

Figure 12. Design steps for a dipole antenna to obtain a desired frequency.

The design process for dipole antenna on a dielectric substrate is illustrated in Figure 12. This technique is comparatively fast and relatively precise. The entire experimental design process took less than five minutes to complete with accuracy of $98 \%$ using a computer with 4 GB RAM and Intel Core 5.

\section{CONCLUSIONS}

This paper presents a simple method of designing an ungrounded dipole antenna from an insulated wire antenna using Hallen's transformation. Four different techniques were analyzed to obtain the most precise technique for obtaining a scaling factor. The surface impedance and dielectric slab solutions had errors in the resonant frequency of approximately $32 \%$. The insulated wire technique offered the best solution for a wide bandwidth with error of $<5.5 \%$ for $f_{c}<3 \mathrm{GHz}$ and $<8 \%$ for $f_{c}=6 \mathrm{GHz}$. A fabricated dipole antenna was scaled using the insulated wire solution. The design frequency lies within $2 \%$ of the measured result and was well within the $-10 \mathrm{~dB}$ impedance bandwidth. The application of this technique to more complicated antennas designs (e.g., meander line antennas) needs further investigation.

\section{REFERENCES}

1. Finkenzeller, D. K., RFID Handbook: Radio-frequency Identification Fundamentals and Applications, 2nd Edition, J. Wiley \& Sons, New York, 2003.

2. Kanesan, M., D. V. Thiel, A. Galehdar, and S. G. O'Keefe, "Rapid analysis and optimization of planar Yagi-Uda dipole arrays printed on a dielectric substrate," Int. J. RF Microwave Comput.Aided Eng., 2013, Doi: 10.1002/mmce.20747.

3. Abbosh, A., "Accurate effective permittivity calculation of printed center-fed dipoles and its application to quasi Yagi-Uda antennas," IEEE Trans. Antennas Propag., Vol. 61, No. 4, 22972300, 2013.

4. Kanesan, M., D. V. Thiel, and S. G. O'Keefe, "The effect of lossy dielectric objects on a UHF RFID meander line antenna," IEEE AP-S International Symposium, 1-2, Chicago, Jul. 2012.

5. Jackson, D. and N. Alexopoulos, "Analysis of planar strip geometries in a substrate-superstrate configuration," IEEE Trans. Antennas Propag., Vol. 34, No. 12, 1430-1438, 1986.

6. Delgado, H. J. and M. H. Thursby, "A novel neural network combined with FDTD for the synthesis of a printed dipole antenna," IEEE Trans. Antennas Propag., Vol. 53, No. 7, 2231-2236, 2005. 
7. Zhang, Y., Z. N. Chen, and M. Y. W. Chia, "Characteristics of planar dipoles printed on finite-size PCBs in UWB radio systems," IEEE AP-S International Symposium, Vol. 3, 2512-2515, Monterey, Jun. 2004.

8. Arima, T., T. Uno, and M. Takahashi "FDTD analysis of printed antenna on thin dielectric sheet including quasi-static approximation," IEEE AP-S International Symposium, Vol. 1, 1022-1025, Monterey, Jun. 2004.

9. Plus, N.-W., User's Manual, Nittany Scientific Inc., 2003.

10. Hallen, E., "Theoretical investigation in the transmitting and receiving qualities of antennae," Nova Acta Regiae Soc. Sci. Upsaliensis, Ser. IV, Vol. 11, 1-44, 1938.

11. Cheng, D. K., Field and Wave Electromagnetics, 2nd Edition, Addison-Wesley, MA, 1983.

12. Collin, R. E., Field Theory of Guided Waves, 2nd Edition, IEEE-Press, NY, 1991.

13. Wait, J. R., Electromagnetic Waves in Stratified Media, IEEE-Press, NY, 1995.

14. Seshadri, S. R., Fundamentals of Transmission Lines and Electromagnetic Fields, Addison-Wesley, MA, 1971.

15. Thiel, D. V. and S. Smith, Switched Parasitic Antennas for Cellular Communications, Artech House, MA, 2002. 